\title{
Neuropathischer Schmerz: Aktualisierung der Leitlinien sinnvoll
}

\section{Hier steht eine Anzeige.}

\author{
Im Rahmen einer Metaanalyse sollte geprüft werden, welche oralen und \\ topischen Medikamente in der Behandlung neuropathischer Schmerzen \\ wirksam sind. Dabei ergaben sich einige praxisrelevante Änderungen.
}

\begin{abstract}
In den letzten 10 Jahren sind zahlreiche Handlungsempfehlungen zur Behandlung neuropathischer Schmerzen oder Schmerzsyndrome gegeben worden. Zwischenzeitlich ist jedoch die Zahl der dazu publizierten Daten und noch laufenden klinischen Studien deutlich angestiegen, sodass eine Aktualisierung dieser Empfehlungen sinnvoll ist, auch vor dem Hintergrund, dass durch die Einführung des Systems GRADE (Grading of Recommendations Assessment, Development, and Evaluation) eine genauere Messung des Evidenzgrades der vorliegenden Daten und Empfehlungen möglich ist.
\end{abstract}

Für diese Metaanalyse und systematischen Review wurden 229 randomisierte doppelblinde placebokontrollierte Studien, in peer-reviewed Journals und unpublizierte Studien von 1966 bis heute eingeschlossen. Als primäre Variable wurde die "number needed to treat" (NNT) für eine mindestens $50 \%$ ige Schmerzreduktion definiert. Die Analyse des Publikationsbias wurde mit 10\% geschätzt. Für die Verwendung von Noradrenalin-Wiederaufnahmehemmern (SNRI, z. B. Duloxetin) wurde eine NNT von 6,4 berechnet, 7,7 für Pregabalin, 7,2 für Gabapentin und 10,6 für topisches Capsaicin. Für Trizyklika, Opioide und lokales Lidocain waren die NNT niedriger. Die Verträglichkeit und Sicherheit war bei topischen Substanzen höher, die Kosten für Trizyklika und Tramadol waren deutlich niedriger.

Basierend auf dieser Metaanalyse empfehlen die Forscher die Verwendung von Trizyklika, SNRI, Pregabalin und Gabapentin als Medikamente für die Erstlinientherapie. Die Verwendung von Lidocain- und Capsaicin-Pflastern sowie Tramadol werden als Zweitlinienoption empfohlen, während für die Verwendung von hochpotenten Opioiden und Botulinumtoxin A lediglich eine schwache Empfehlung gegeben wird.
Fazit: Die Forscher raten aufgrund der erhobenen Daten in dieser Metaanalyse zu einer Aktualisierung der bisherigen Leitlinien zur Behandlung neuropathischer Schmerzen.

Tim Hagenacker

Finnerup NB et al. Pharmacotherapy of neuropathic pain in adults: a systematic review and meta-analysis. Lancet Neurol. 2015;14(2):162-73.

\section{Kommentar von PD Dr. med.} Hagenacker:

Diese Metaanalyse unterstreicht, dass eine Aktualisierung der Leitlinien zur Behandlung neuropathischer Schmerzen nun vorgenommen werden sollte. Die Analyse stützt sich dabei vornehmlich auf den wesentlichsten Parameter der Therapie, nämlich die Schmerzreduktion, ohne die Sicherheit und Verträglichkeit und letztlich auch ökonomischen Parameter zu vernachlässigen. Wenngleich sich aus dieser Metaanalyse zwar keine bahnbrechenden Änderungen der bisherigen medikamentösen Behandlung neuropathischer Schmerzen ableiten lassen, ist eine Überarbeitung der Leitlinien nichtsdestotrotz sinnvoll, weil sich im Detail doch durchaus Änderungen zeigen, die für die Behandlung des einzelnen Patienten in der Praxis wichtig sind. Der einzige Stolperstein dieser Metaanalyse ist, dass alle Entitäten, die sich hinter neuropathischen Schmerzen verbergen, in einer Analyse vereinheitlicht wurden, wenngleich sich die pathophysiologischen Mechanismen und die daraus abzuleitenden Therapiemaßnahmen unterscheiden können. Dieser Punkt wird aber weiterhin diskutiert und muss sicherlich im Rahmen eines Updates der Leitlinien berücksichtigt werden.

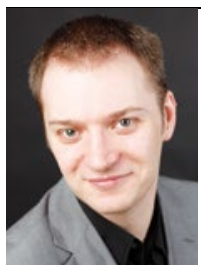

PD Dr. med. Tim Hagenacker Klinik für Neurologie, Universitätsklinikum Essen tim.hagenacker@ukessen.de 